\title{
THE EQUAL SPLIT-OFF SET FOR COOPERATIVE GAMES
}

\author{
RODICA BRANZEI \\ Faculty of Computer Science, "Alexandru Ioan Cuza" University, 700483 Iaşi, Romania \\ E-mail: branzei@infoiasi.ro \\ DINKO DIMITROV \\ Institute of Mathematical Economics, Bielefeld University, 33501 Bielefeld, Germany \\ E-mail:d.dimitrov@wiwi.uni-bielefeld.de \\ STEF TIJS \\ Faculty of Economics and Business Administration \\ Tilburg University, 5000 LE Tilburg, The Netherlands \\ E-mail:S.H.Tijs@uvt.nl \\ and \\ Department of Mathematics, University of Genoa, Italy
}

\begin{abstract}
In this paper the equal split-off set is introduced as a new solution concept for cooperative games. This solution is based on egalitarian considerations and it turns out that for superadditive games the equal split-off set is a subset of the equal division core. Moreover, the proposed solution is single valued on the class of convex games and it coincides with the Dutta-Ray constrained egalitarian solution.
\end{abstract}

1. Introduction. In this paper we propose a new set valued solution concept for cooperative games with transferable utility that we call the equal split-off set. This solution is based on egalitarian considerations and it is inspired by the Dutta-Ray algorithm for finding the constrained egalitarian solution for convex games (cf. Dutta and Ray (1989)).

More precisely, we consider a world $N$ of $n$ players, $N=\{1, \ldots, n\}$, who believe in equal share cooperation. For each coalition $S \subseteq N$, let the real number $v(S)$ represent

Journal of Economic Literature Classification: C71.

Key words and phrases: convex games, egalitarianism, equal split-off set, superadditive games.

We would like to thank an anonymous referee for her/his valuable comments and suggestions that helped us to shape more precisely the focus of the paper. D. Dimitrov gratefully acknowledges financial support from the Alexander von Humboldt Foundation.

The paper is in final form and no version of it will be published elsewhere. 
what the players in $S$ can get if they cooperate (i.e. $v(S)$ is the worth or the value of coalition $S$ ). We assume that the entire set of players will cooperate and deal with the question how the whole amount of money $v(N)$ generated by $N$ should be divided among the players by considering the following step-wise process.

First, one of the coalitions with maximal average worth, say $T_{1}$, forms and the players in $T_{1}$ divide equally the worth $v\left(T_{1}\right)$. In step 2 one of the coalitions in $N \backslash T_{1}$ with maximal average marginal worth w.r.t. $T_{1}$, say $T_{2}$, forms, joins costless $T_{1}$, and divides equally the increase in value $v\left(T_{2} \cup T_{1}\right)-v\left(T_{1}\right)$ among its members. The process stops when a partition of $N$ of the form $\left\langle T_{1}, \ldots, T_{K}\right\rangle$ for some $1 \leq K \leq n$ is reached. This procedure generates an efficient payoff vector $x \in \mathbb{R}^{n}$ which we call an equal split-off allocation. The equal split-off set is then defined as consisting of all equal split-off allocations.

Notice that the difference between the above procedure and the Dutta-Ray algorithm for finding the constrained egalitarian solution for convex games is that the corresponding selected coalitions need not be the largest coalitions with the highest average worth.

The outline of this paper is as follows. After some preliminaries in Section 2, we introduce the equal split-off set for arbitrary TU-games in Section 3 and exemplify it. We consider in Section 4 the class of superadditive games and show that, on this class, any allocation in the equal split-off set belongs to the equal division core of the corresponding game and, consequently, it is individually rational. In Section 5 we concentrate on the class of convex games and prove that the equal split-off set of a convex game consists of a unique allocation which is the constrained egalitarian solution of that game. We conclude in Section 6 with some final remarks.

2. Preliminaries. A TU-game is a pair $(N, v)$, where $N=\{1, \ldots, n\}$ is a set of players and $v: 2^{N} \rightarrow \mathbb{R}$ is a characteristic function on $N$ satisfying $v(\emptyset)=0$. Often, we will identify a game $(N, v)$ with its characteristic function $v$. For any coalition $S \subseteq N, v(S)$ is the worth of coalition $S$, i.e. the members of $S$ can obtain a total payoff of $v(S)$ by agreeing to cooperate.

A game $v$ is called

- superadditive, if $v(S \cup T) \geq v(S)+v(T)$ for all $S, T \subseteq N$ with $S \cap T=\emptyset$;

- convex, if $v(S \cup T)+v(S \cap T) \geq v(S)+v(T)$ for all $S, T \subseteq N$.

An allocation in a game $v$ is a payoff vector $x \in \mathbb{R}^{n}$. An allocation of $v(N)$ such that this amount is cleared is called efficient, and an allocation $x$ such that $x_{i} \geq v(i)$ for each $i \in N$ is called individually rational. The imputation set $I(v)$ of a game $v$ is the set of all efficient and individually rational allocations, i.e.

$$
I(v)=\left\{x \in \mathbb{R}^{n} \mid \sum_{i \in N} x_{i}=v(N) \text { and } x_{i} \geq v(i) \text { for each } i \in N\right\} .
$$

Further, an allocation is called stable if any coalition $S \subseteq N$ receives at least its value $v(S)$. The core $C(v)$ of a game $v$ is the set of all efficient and stable allocations (Gillies, $1953)$, i.e. the set

$$
C(v)=\left\{x \in I(v) \mid \sum_{i \in S} x_{i} \geq v(S) \text { for each } S \in 2^{N}\right\} .
$$


For each game $v$ we have that $C(v)$ is a subset of the equal division core $E D C(v)$ of $v$. The latter concept was introduced by Selten (1972) as the set

$$
\left\{x \in I(v) \mid \nexists S \in 2^{N} \backslash\{\emptyset\} \text { s.t. } \frac{v(S)}{|S|}>x_{i} \text { for all } i \in S\right\}
$$

consisting of all efficient payoff vectors which cannot be improved upon by the equal division allocation of any subcoalition.

An interesting element of the core $C(v)$ of a convex game $v$ (and, hence, of $E D C(v)$ ) is the Dutta-Ray constrained egalitarian solution $D R(v)$. This solution consists of the unique allocation in $C(v)$ that Lorenz dominates every other core allocation. In their seminal paper, Dutta and Ray (1989) provide an algorithm for generating $D R(v)$ for each convex game $v$. We apply a modified version of the Dutta-Ray algorithm to any TU-game in order to produce allocations in the equal split-off set of $v$.

3. The equal split-off set. Let $v$ be an arbitrary TU-game and $\pi=\left\langle T_{1}, \ldots, T_{K}\right\rangle$ be an ordered partition of the player set $N$. We set $v_{1}:=v$, and for each $k \in\{2, \ldots, K\}$ we define the marginal game

$$
v_{k}: 2^{N \backslash\left(\cup_{s=1}^{k-1} T_{s}\right)} \rightarrow \mathbb{R}
$$

by

$$
v_{k}(S):=v_{k-1}\left(T_{k-1} \cup S\right)-v_{k-1}\left(T_{k-1}\right)=v\left(\left(\cup_{s=1}^{k-1} T_{s}\right) \cup S\right)-v\left(\cup_{s=1}^{k-1} T_{s}\right) .
$$

We call the partition $\pi=\left\langle T_{1}, \ldots, T_{K}\right\rangle$ of $N$ a suitable ordered partition with respect

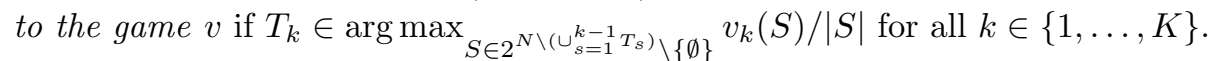

Given such a partition $\pi$, the equal split-off allocation for $v$ generated by $\pi$ is the efficient payoff vector $x=\left(x_{i}\right)_{i \in N} \in \mathbb{R}^{n}$, where for all $T_{k} \in \pi$ and all $i \in T_{k}, x_{i}=$ $v_{k}\left(T_{k}\right) /\left|T_{k}\right|$.

Now we define the equal split-off set $\operatorname{ESOS}(v)$ of the game $v$ as the set

$\left\{x \in \mathbb{R}^{n} \mid \exists \pi\right.$ s.t. $x$ is an equal split-off allocation for $v$ generated by $\left.\pi\right\}$.

In order to illustrate this solution concept, let us have a look at the following examples:

EXAMPLE 1 (2-person superadditive games). Let $v$ be a game on the player set $N=\{1,2\}$ satisfying $v(1,2) \geq v(1)+v(2)$. Suppose without loss of generality that $v(1) \geq v(2)$ and consider the following four cases:

(i) $v(1)>\frac{1}{2} v(1,2)$. Then $\langle\{1\},\{2\}\rangle$ is the unique suitable ordered partition and $E S O S(v)$ $=\{(v(1), v(1,2)-v(1))\}$;

(ii) $v(2)<v(1)=\frac{1}{2} v(1,2)$. In this case $\operatorname{ESOS}(v)=\left\{\left(\frac{1}{2} v(1,2), \frac{1}{2} v(1,2)\right)\right\}$ corresponding to the suitable ordered partitions $\langle\{1\},\{2\}\rangle$ and $\langle\{1,2\}\rangle$;

(iii) $v(2)=v(1)=\frac{1}{2} v(1,2)$. Also here $\operatorname{ESOS}(v)=\left\{\left(\frac{1}{2} v(1,2), \frac{1}{2} v(1,2)\right)\right\}=\{(v(1), v(2))\}$ corresponding to the three suitable ordered partitions $\langle\{1\},\{2\}\rangle,\langle\{2\},\{1\}\rangle$, and $\langle\{1,2\}\rangle$; (iv) $v(1)<\frac{1}{2} v(1,2)$. Then $\langle\{1,2\}\rangle$ is the unique suitable ordered partition and $\operatorname{ESOS(v)}$ $=\left\{\left(\frac{1}{2} v(1,2), \frac{1}{2} v(1,2)\right)\right\}$.

EXAmple 2 (Simple games). In a simple game $v$ on player set $N$ we have that for all $S \subseteq N, v(S) \in\{0,1\}$ with $v(\emptyset)=0$ and $v(N)=1$. A coalition $S \subseteq N$ is called minimal winning if $v(S)=1$ and $v\left(S^{\prime}\right)=0$ for all $S^{\prime} \subset S \subseteq N$. Given a simple game $v$, we denote 
the set of all minimal winning coalitions with a smallest cardinality by $W^{s}$. In the case of simple games $\operatorname{ESOS}(v)=\left\{(1 /|S|) e^{S} \mid S \in W^{s}\right\}$ because for any suitable ordered partition $\left\langle T_{1}, \ldots, T_{K}\right\rangle$ we will have $T_{1} \in W^{s}$, and all players in $T_{1}$ will receive $1 /\left|T_{1}\right|$ whereas the players in $N \backslash T_{1}$ will receive payoff 0 .

ExAmple 3 (Glove games). Let $N=L \cup R, L \cap R \neq \emptyset$ and the game $v$ be defined by $v(S)=\min \{|S \cap L|,|R \cap S|\}$ for each $S \subseteq N$. If $|L|=|R|$, then $\operatorname{ESOS}(v)=\left\{\left(\frac{1}{2}, \ldots, \frac{1}{2}\right)\right\}$ that can be generated by many suitable ordered partitions, where each element $T_{k}$ of such a partition has the property that $\left|T_{k} \cap L\right|=\left|T_{k} \cap R\right|$. In case $|L|>|R|$ each element $x \in \operatorname{ESOS}(v)$ satisfies $x_{i}=\frac{1}{2}$ for each $i \in R$ and for $|R|$ elements of $L$, and $x_{i}=0$ for the other elements of $L$. Conversely, all elements of this type belong to $\operatorname{ESOS}(v)$.

EXAMPLE 4 (A 2-person non-superadditive game). Let $v$ be a game on the player set $N=\{1,2\}$ satisfying $v(\emptyset)=v(1,2)=0, v(1)=3$, and $v(2)=2$. Then $\langle\{1\},\{2\}\rangle$ is the unique suitable ordered partition and $\operatorname{ESOS}(v)=\{(3,-3)\}$.

One can easily check that the suitable ordered partitions generating equal split-off allocations in Examples 1-4 satisfy a monotonicity property w.r.t. average worth as stated in

Proposition 1. Let $v$ be an arbitrary $T U$-game and let $\left\langle T_{1}, \ldots, T_{K}\right\rangle$ be a suitable ordered partition of $N$ w.r.t. $v$. Then

$$
\max _{S \in 2^{N \backslash\left(\cup_{s=1}^{k-1} T_{s}\right)} \backslash\{\emptyset\}} \frac{v_{k}(S)}{|S|} \geq \max _{S \in 2^{N \backslash\left(\cup_{s=1}^{k} T_{s}\right)} \backslash\{\emptyset\}} \frac{v_{k+1}(S)}{|S|}
$$

for all $k \in\{1, \ldots, K-1\}$.

Proof. By definition of $T_{k}$ we have

$$
\frac{v\left(\cup_{s=1}^{k} T_{s}\right)-v\left(\cup_{s=1}^{k-1} T_{s}\right)}{\left|T_{k}\right|} \geq \frac{v\left(\cup_{s=1}^{k+1} T_{s}\right)-v\left(\cup_{s=1}^{k-1} T_{s}\right)}{\left|T_{k}\right|+\left|T_{k+1}\right|} .
$$

Moreover,

$$
\frac{v\left(\cup_{s=1}^{k+1} T_{s}\right)-v\left(\cup_{s=1}^{k-1} T_{s}\right)}{\left|T_{k}\right|+\left|T_{k+1}\right|}=\frac{v\left(\cup_{s=1}^{k+1} T_{s}\right)-v\left(\cup_{s=1}^{k} T_{s}\right)+v\left(\cup_{s=1}^{k} T_{s}\right)-v\left(\cup_{s=1}^{k-1} T_{s}\right)}{\left|T_{k}\right|+\left|T_{k+1}\right|},
$$

implying that

$$
\frac{v\left(\cup_{s=1}^{k} T_{s}\right)-v\left(\cup_{s=1}^{k-1} T_{s}\right)}{\left|T_{k}\right|} \geq \frac{v\left(\cup_{s=1}^{k+1} T_{s}\right)-v\left(\cup_{s=1}^{k} T_{s}\right)+v\left(\cup_{s=1}^{k} T_{s}\right)-v\left(\cup_{s=1}^{k-1} T_{s}\right)}{\left|T_{k}\right|+\left|T_{k+1}\right|} .
$$

This inequality is equivalent to

$$
\begin{aligned}
\left(v\left(\cup_{s=1}^{k} T_{s}\right)-v\right. & \left.\left(\cup_{s=1}^{k-1} T_{s}\right)\right)\left|T_{k}\right|+\left(v\left(\cup_{s=1}^{k} T_{s}\right)-v\left(\cup_{s=1}^{k-1} T_{s}\right)\right)\left|T_{k+1}\right| \\
& \geq\left(v\left(\cup_{s=1}^{k+1} T_{s}\right)-v\left(\cup_{s=1}^{k} T_{s}\right)\right)\left|T_{k}\right|+\left(v\left(\cup_{s=1}^{k} T_{s}\right)-v\left(\cup_{s=1}^{k-1} T_{s}\right)\right)\left|T_{k}\right|,
\end{aligned}
$$

which is in turn equivalent to

$$
\left(v\left(\cup_{s=1}^{k} T_{s}\right)-v\left(\cup_{s=1}^{k-1} T_{s}\right)\right)\left|T_{k+1}\right| \geq\left(v\left(\cup_{s=1}^{k+1} T_{s}\right)-v\left(\cup_{s=1}^{k} T_{s}\right)\right)\left|T_{k}\right| .
$$

We refer the reader to Yanovskaya (2005) for an axiomatic characterization of the equal split-off set on the class of arbitrary TU-games that uses a consistency property à la Hart-Mas-Colell (cf. Hart and Mas-Colell (1989)). 
4. The equal split-off set for superadditive games. We consider now an interesting additional property of our solution concept on the class of superadditive games. As it turns out, the equal split-off set of a superadditive game is a refinement of the equal division core of that game.

THEOREM 1. Let $v$ be a superadditive game. Then $\operatorname{ESOS}(v) \subseteq E D C(v)$.

Proof. Let $x \in \operatorname{ESOS}(v)$ be generated by the suitable ordered partition $\left\langle T_{1}, \ldots, T_{K}\right\rangle$. Take $S \in 2^{N} \backslash\{\emptyset\}$. We have to prove that there is $i \in S$ such that $x_{i} \geq v(S) /|S|$.

Let $m \in\{1, \ldots, K\}$ be the smallest number such that $T_{m} \cap S \neq \emptyset$. Then

$$
\begin{aligned}
\frac{v(S)}{|S|} & \leq \frac{v\left(\left(\cup_{s=1}^{m-1} T_{s}\right) \cup S\right)-v\left(\cup_{s=1}^{m-1} T_{s}\right)}{|S|} \\
& \leq \frac{v\left(\cup_{s=1}^{m} T_{s}\right)-v\left(\cup_{s=1}^{m-1} T_{s}\right)}{\left|T_{m}\right|} \\
& =\frac{v_{m}\left(T_{m}\right)}{\left|T_{m}\right|}=\max _{T \in 2^{N \backslash\left(\cup_{s=1}^{m-1} T_{s}\right)} \backslash\{\emptyset\}} \frac{v_{m}(T)}{|T|},
\end{aligned}
$$

where the first inequality follows from the superadditivity of $v$ and the second inequality from the definition of $T_{m}$. Note that

$$
x_{i}=\max _{T \in 2^{N \backslash\left(\cup_{s=1}^{m-1} T_{s}\right) \backslash\{\emptyset\}}} \frac{v_{m}(T)}{|T|} \geq \frac{v(S)}{|S|}
$$

for each $i \in T_{m} \cap S$. So, $x \in E D C(v)$ implying that $E S O S(v) \subseteq E D C(v)$.

The next example provides a game for which the equal split-off set is a strict subset of the equal division core.

ExAmple 5. Let $N=\{1,2,3\}$ and $v$ be a glove game with $L=\{1,2\}$ and $R=\{3\}$. Then $E D C(v)=\left\{x \in I(v) \mid x_{3} \geq \frac{1}{2}\right\}$ and $\operatorname{ESOS}(v)=\left\{\left(\frac{1}{2}, 0, \frac{1}{2}\right),\left(0, \frac{1}{2}, \frac{1}{2}\right)\right\}$.

REMARK 1. Clearly, by Theorem 1, each equal split-off allocation for a superadditive game is individually rational. As illustrated in Example 4, this need not be the case for non-superadditive games.

5. The equal split-off set for convex games. We show in this section that the equal split-off set of a convex game consists of a single allocation which is the Dutta-Ray egalitarian solution of that game.

Let $\left\langle D_{1}, \ldots, D_{P}\right\rangle$ be the ordered partition of $N$ according to the Dutta-Ray algorithm for finding the constrained egalitarian solution $D R(v)$ of a convex game $v$. In each step $p \in\{1, \ldots, P\}$ of the Dutta-Ray algorithm, the coalition $D_{p}$ is the largest element in the set

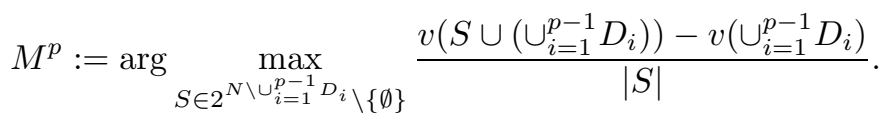

We recall that for each $p \in\{1, \ldots, P\}$ the set $M^{p}$ has a lattice structure w.r.t. the partial ordering of inclusion. So,

$$
D_{p}=\cup\left\{D \mid D \in M^{p}\right\}
$$


For further use, we let

$$
d_{p}:=\frac{v\left(D_{p} \cup\left(\cup_{r=1}^{p-1} D_{r}\right)\right)-v\left(\cup_{r=1}^{p-1} D_{r}\right)}{\left|D_{p}\right|} \quad \text { for each } p \in\{1, \ldots, P\} .
$$

Suppose now that we are given a convex game $v$, its ordered partition $\left\langle D_{1}, \ldots, D_{P}\right\rangle$ according to the Dutta-Ray algorithm, and an allocation $x=\left(x_{i}\right)_{i \in N}$ in the equal split-off set $\operatorname{ESOS}(v)$ of $v$ that is generated by the suitable ordered partition $\left\langle T_{1}, \ldots, T_{K}\right\rangle$. Then we have the following

LEMma 1. Let $v_{1}\left(T_{1}\right) /\left|T_{1}\right|=a_{1}$ and $k_{1} \in\{1, \ldots, K\}$ be the largest number for which $v_{k_{1}}\left(T_{k_{1}}\right) /\left|T_{k_{1}}\right|=a_{1}$. Then $a_{1}=d_{1}$ and $D_{1}=\cup_{j=1}^{k_{1}} T_{j}$.

Proof. Since $T_{1} \in M^{1}$ and $D_{1}=\cup\left\{D \mid D \in M^{1}\right\}$, we have $T_{1} \subseteq D_{1}$ and $a_{1}=$ $v_{1}\left(T_{1}\right) /\left|T_{1}\right|=v\left(T_{1}\right) /\left|T_{1}\right|=d_{1}$.

Next we show that $\cup_{j=1}^{k_{1}} T_{j} \subseteq D_{1}$ by proving by induction that $\cup_{j=1}^{k^{\prime}} T_{j} \subseteq D_{1}$ for each $k^{\prime} \in\left\{1, \ldots, k_{1}\right\}$.

For $k^{\prime}=1$ the inclusion is correct.

Suppose that for some $k^{\prime} \in\left\{1, \ldots, k_{1}-1\right\}$ we have $\cup_{j=1}^{k^{\prime}} T_{j} \subseteq D_{1}$. We show that $\cup_{j=1}^{k^{\prime}+1} T_{j} \subseteq D_{1}$. We have

$$
\begin{aligned}
d_{1}\left|T_{k^{\prime}+1}\right| & =v_{k^{\prime}+1}\left(T_{k^{\prime}+1}\right)=v_{1}\left(\cup_{j=1}^{k^{\prime}+1} T_{j}\right)-v_{1}\left(\cup_{j=1}^{k^{\prime}} T_{j}\right) \\
& =v_{1}\left(\cup_{j=1}^{k^{\prime}+1} T_{j}\right)-d_{1}\left|\cup_{j=1}^{k^{\prime}} T_{j}\right|,
\end{aligned}
$$

i.e.,

$$
d_{1}\left|\cup_{j=1}^{k^{\prime}+1} T_{j}\right|=v_{1}\left(\cup_{j=1}^{k^{\prime}+1} T_{j}\right),
$$

implying that $\cup_{j=1}^{k^{\prime}+1} T_{j} \subseteq D_{1}$. Hence, $\cup_{j=1}^{k_{1}} T_{j} \subseteq D_{1}$.

Next we prove that $\cup_{j=1}^{k_{1}} T_{j}=D_{1}$. Suppose that we have $V=D_{1} \backslash \cup_{j=1}^{k_{1}} T_{j} \neq \emptyset$. First, from the selection of $k_{1}$ and Proposition 1 it follows that

$$
\frac{v_{k_{1}+1}(S)}{|S|}<a_{1}=d_{1} \quad \text { for each } S \in 2^{N \backslash \cup_{j=1}^{k_{1}} T_{j}} \backslash\{\emptyset\},
$$

implying

$$
\frac{v_{k_{1}+1}(V)}{|V|}<d_{1}
$$

On the other hand,

$$
\begin{aligned}
\frac{v_{k_{1}+1}(V)}{|V|} & =\frac{v_{1}\left(D_{1}\right)-v_{1}\left(\cup_{j=1}^{k_{1}} T_{j}\right)}{|V|} \\
& =\frac{d_{1}\left|D_{1}\right|-d_{1}\left|\left(\cup_{j=1}^{k_{1}} T_{j}\right)\right|}{|V|}=\frac{d_{1}|V|}{|V|} \\
& =d_{1}
\end{aligned}
$$

which contradicts (1). Hence, we have proved that $\cup_{j=1}^{k_{1}} T_{j}=D_{1}$.

Theorem 2. Let $v$ be a convex game. Then $\operatorname{ESOS}(v)=\{D R(v)\}$.

Proof. Let $\left\langle D_{1}, \ldots, D_{P}\right\rangle$ be the ordered partition of $N$ according to the Dutta-Ray algorithm for finding the constrained egalitarian solution $D R(v)=\left(D R_{i}(v)\right)_{i \in N}$ of $v$. 
Take an arbitrary allocation $x=\left(x_{i}\right)_{i \in N}$ in the equal split-off set $\operatorname{ESOS}(v)$ of $v$, and let it be generated by the suitable ordered partition $\left\langle T_{1}, \ldots, T_{K}\right\rangle$.

We show by induction on $p \in\{1, \ldots, P\}$ that there exist $k_{1}^{*}, \ldots, k_{p}^{*}, \ldots, k_{P}^{*} \in\{1, \ldots, K\}$ with $1 \leq k_{1}^{*}<\ldots<k_{p}^{*}<\ldots<k_{P}^{*} \leq K$ such that for each $p \in\{1, \ldots, P\}$ and each $j \in\left\{k_{p-1}^{*}+1, \ldots, k_{p}^{*}\right\}$ we have

$$
\cup_{j=k_{p-1}^{*}+1}^{k_{p}^{*}} T_{j}=D_{p} \text { and } \frac{v_{j}\left(T_{j}\right)}{\left|T_{j}\right|}=d_{p} .
$$

For $p=1$ let $k_{1}^{*}=k_{1}$, where $k_{1} \in\{1, \ldots, K\}$ is the largest number for which $v_{k_{1}}\left(T_{k_{1}}\right) /\left|T_{k_{1}}\right|=a_{1}=v_{1}\left(T_{1}\right) /\left|T_{1}\right|$. By Lemma 1 we have $\cup_{j=1}^{k_{1}} T_{j}=D_{1}$ and $v_{j}\left(T_{j}\right) /\left|T_{j}\right|=$ $d_{1}$ for each $j \in\left\{1, \ldots, k_{1}^{*}\right\}$.

Suppose that for some $p \in\{1, \ldots, P-1\}$ there exists $k_{p}^{*}$ such that $k_{p-1}^{*}<k_{p}^{*}<K$ for which (2) holds. We show that there exists $k_{p+1}^{*}$, such that $k_{p}^{*}<k_{p+1}^{*} \leq K$, for which $\cup_{j=k_{p}^{*}+1}^{k_{p+1}^{*}} T_{j}=D_{p+1}$ and $v_{j}\left(T_{j}\right) /\left|T_{j}\right|=d_{p+1}$ for each $j \in\left\{k_{p}^{*}+1, \ldots, k_{p+1}^{*}\right\}$. by

Notice that $\cup_{j=1}^{k_{p}^{*}} T_{j}=\cup_{l=1}^{p} D_{l}$ implying that the game $v_{k_{p}^{*}+1}: 2^{N \backslash \cup_{j=1}^{k_{p}^{*}} T_{j}} \rightarrow \mathbb{R}$ defined

$$
v_{k_{p}^{*}+1}(S):=v\left(\left(\cup_{j=1}^{k_{p}^{*}} T_{j}\right) \cup S\right)-v\left(\cup_{j=1}^{k_{p}^{*}} T_{j}\right)
$$

and the game $v_{p+1}: 2^{N \backslash \cup_{l=1}^{p} D_{l}} \rightarrow \mathbb{R}$ defined by

$$
v_{p+1}(S):=v\left(\left(\cup_{l=1}^{p} D_{l}\right) \cup S\right)-v\left(\cup_{l=1}^{p} D_{l}\right)
$$

coincide.

Let $a_{k_{p}^{*}+1}=v_{k_{p}^{*}+1}\left(T_{k_{p}^{*}+1}\right) /\left|T_{k_{p}^{*}+1}\right|$ and $k_{p+1} \in\left\{k_{p}^{*}+1, \ldots, K\right\}$ be the largest number for which $v_{k_{p+1}}\left(T_{k_{p+1}}\right) /\left|T_{k_{p+1}}\right|=a_{k_{p}^{*}+1}$. Take $k_{p+1}^{*}=k_{p+1}$. Given the coincidence of the games $v_{k_{p}^{*}+1}$ and $v_{p+1}$ and their convexity (cf. Dutta and Ray (1991)), we can apply the same argument as in Lemma 1 to conclude that (2) holds also for $p+1$.

It follows then that the suitable ordered partition $\left\langle T_{1}, \ldots, T_{K}\right\rangle$ is a refinement of $\left\langle D_{1}, \ldots, D_{P}\right\rangle$ of the form

$$
\left\langle\left\langle T_{1}, \ldots, T_{k_{1}^{*}}\right\rangle,\left\langle T_{k_{1}^{*}+1}, \ldots, T_{k_{2}^{*}}\right\rangle, \ldots,\left\langle T_{k_{P-1}^{*}+1}, \ldots, T_{k_{P}^{*}}\right\rangle\right\rangle
$$

with $T_{k_{P}^{*}}=T_{K}$, and for each partition $\left\langle T_{k_{p-1}^{*}+1}, \ldots, T_{k_{p}^{*}}\right\rangle$ of $D_{p}, p \in\{1, \ldots, P\}$, the members of each element $T_{k}, k \in\left\{k_{p-1}^{*}+1, \ldots, k_{p}^{*}\right\}$ with $k_{0}^{*}=0$, receive the same payoff $d_{p}$. Thus, we have $x=D R(v)$ implying that $\operatorname{ESOS}(v)=\{D R(v)\}$ for a convex game $v$.

6. Concluding remarks. In this paper the equal split-off set has been introduced for arbitrary TU-games as a new set valued solution concept based on egalitarian considerations. We have proved that for any superadditive game the new solution concept is a refinement of the equal division core. Moreover, we have shown that for each convex game the equal split-off set consists of a single allocation which is the Dutta-Ray constrained egalitarian solution for that game. Recently, an axiomatic characterization of this solution on the class of arbitrary TU-games has been provided by Yanovskaya (2005). Further investigations could be done for clarifying possible relations between the equal split-off set and existing egalitarianism-based solution concepts for arbitrary TU-games such as the strong-constrained egalitarian allocations (cf. Dutta and Ray (1991)), the egalitarian 
set, the preegalitarian set and the stable egalitarian set (cf. Arin and Inarra (2002)) and for balanced games like the Lorenz solution (cf. Hougaard et al. (2001)), the Lorenz stable set and the egalitarian core (cf. Arin and Inarra (2001)).

\section{References}

[1] J. Arin and E. Inarra, Egalitarian sets for TU-games, International Game Theory Review 4 (2002), 183-199.

[2] J. Arin and E. Inarra, Egalitarian solutions in the core, International Journal of Game Theory 30 (2001), 187-193.

[3] B. Dutta and D. Ray, Constrained egalitarian allocations, Games and Economic Behavior 3 (1991), 403-422.

[4] B. Dutta and D. Ray, A concept of egalitarianism under participation constraints, Econometrica 57 (1989), 615-635.

[5] D. B. Gillies, Some theorems on n-person games, Ph.D. Thesis, Princeton University Press, Princeton, New Jersey, 1953.

[6] S. Hart and A. Mas-Colell, Potential, value, and consistency, Econometrica 57 (1989), 589-614.

[7] J. Hougaard, B. Peleg and L. Thorlund-Petersen, On the set of Lorenz-maximal imputations in the core of a balanced game, International Journal of Game Theory 30 (2001), 147-165.

[8] R. Selten, Equal share analysis of characteristic function experiments, in: H. Sauermann (ed.), Contributions to Experimentation in Economics. Vol. 3, Mohr, Tübingen, 1972, 130165.

[9] E. Yanovskaya, Consistency of the egalitarian solution for the class of arbitrary TU-games, Mimeo, St. Petersburg Institute for Economics and Mathematics, 2005. 